PROCEEDINGS OF THE

AMERICAN MATHEMATICAL SOCIETY

Volume 132, Number 12, Pages 3569-3576

S 0002-9939(04)07585-9

Article electronically published on July 22, 2004

\title{
ON LOCAL REPRESENTATIONS OF VON NEUMANN ALGEBRAS
}

\author{
FLORIN POP
}

(Communicated by David R. Larson)

\begin{abstract}
We prove that every bounded, linear, 2-local Hilbert space representation of a von Neumann algebra is a representation. In contrast, 1-local representations may fail to be multiplicative, even at the 2 by 2 matrix algebra level.
\end{abstract}

Cohomology and representations have long been central to the understanding of the fine structure of von Neumann algebras. A derivation of a von Neumann algebra $M$ into a dual, normal $M$-bimodule $X$ is a linear map $\delta: M \rightarrow X$ satisfying $\delta(x y)=\delta(x) y+x \delta(y)$, for all $x, y \in M$. Kadison [7] and Sakai [16] proved that every derivation $\delta: M \rightarrow M$ is inner, that is, $\delta(x)=a x-x a$ for some $a \in M$. The situation is quite different if $X=B(H)$. These derivations are known to be implemented by operators $a \in B(H)$ for all von Neumann algebras except the finite ones, for which, despite significant progress, the problem remains open. Remarkably, Kirchberg 10] showed that the derivation (into $B(H)$ ) problem is equivalent to the celebrated similarity problem: is every bounded representation of a $C^{*}$-algebra similar to a *-representation?

Kadison [8] and Larson and Sourour [11 introduced local derivations. These are linear maps $\theta: M \rightarrow X$ such that, for every $a \in M$, there exists a derivation $\delta_{a}: M \rightarrow X$ satisfying $\theta(a)=\delta_{a}(a)$. Kadison [8] proved that every local derivation is a derivation. Building upon Larson and Sourour's results, Brešar and Šemrl [2] proved that, for separable Hilbert spaces $H$, every linear local automorphism of $B(H)$ is an automorphism. Šemrl [17] removed the linearity assumptions and was led to introduce 2-local derivations and 2-local automorphisms. He proved that, in the separable Hilbert space case, 2-local automorphisms (derivations) of $B(H)$ are automorphisms (derivations), no assumption on linearity, surjectivity or continuity being required. For other related results we refer to [1], [2], [3], [4], [6], [11], [12], [13], [17] and the references therein.

This paper originated in our attempt to investigate whether local representations of von Neumann algebras are representations. This was a natural thing to ask, in view of Kadison's and Kirchberg's results. While our focus was on linear maps, 2-local representations (so far relevant only in the absence of linearity) were to play a surprisingly important role. Our main result is that every bounded, linear 2-local representation of a von Neumann algebra into $B(H)$ is a representation. In contrast to this situation, 1-local representations are multiplicative only on abelian algebras.

Received by the editors February 3, 2003 and, in revised form, May 22, 2003.

2000 Mathematics Subject Classification. Primary 46L10, 46K10. 
In section 2 we present an example of a local representation of the algebra of 2 by 2 matrices that fails to be a representation. We conclude with an application to 2-local automorphisms.

\section{LOCAL REPRESENTATIONS}

In this section we begin the investigation of local representations, with the goal of going as far as possible within the 1-local property.

1.1. Definition. Let $M$ be a von Neumann algebra. A bounded, linear map $\phi$ : $M \rightarrow B(H)$ is called a local (or 1-local) representation if, for every $x \in M$, there exists a bounded representation $\pi_{x}: M \rightarrow B(H)$ satisfying $\phi(x)=\pi_{x}(x)$. (It is not assumed that $M$ and $B(H)$ have the same identity.)

1.2. Remark. Let $e \in M$ be an idempotent $\left(e^{2}=e\right)$. Then $\phi(e)=\pi_{e}(e)=\pi_{e}\left(e^{2}\right)=$ $\pi_{e}(e)^{2}=\phi(e)^{2}$, which shows that $\phi(e)$ is an idempotent as well.

1.3. Lemma. Let $e$ and $f$ be idempotents in $B(H)$ such that $e f+f e=0$. Then ef $=f e=0$.

Proof. We have $0=e(e f+f e)=e f+e f e$ and $0=(e f+f e) e=e f e+f e$; hence ef $=f e$, which implies $e f=f e=0$.

1.4. Proposition. If $M$ is an abelian von Neumann algebra, then every local representation $\phi: M \rightarrow B(H)$ is multiplicative, therefore is a representation.

Proof. Let $p$ and $q$ be mutually orthogonal (self-adjoint) projections in $M$. Then $p+q$ is a projection and, by 1.2 , we have $\phi(p)+\phi(q)=\phi(p+q)=\phi(p+q)^{2}=$ $(\phi(p)+\phi(q))^{2}=\phi(p)^{2}+\phi(q)^{2}+\phi(p) \phi(q)+\phi(q) \phi(p)$, which shows that $\phi(p) \phi(q)+$ $\phi(q) \phi(p)=0$. Now 1.3 implies that $\phi(p) \phi(q)=0$.

Let $p$ and $q$ be commuting projections in $M$. Then $p=p_{1}+r$ and $q=q_{1}+r$, where $p_{1}, q_{1}$, and $r$ are mutually orthogonal projections. From the first part of the proof we have $\phi(p) \phi(q)=\left(\phi\left(p_{1}\right)+\phi(r)\right)\left(\phi\left(q_{1}\right)+\phi(r)\right)=\phi(r)^{2}=\phi(r)=\phi(p q)$. Since every von Neumann algebra is equal to the norm-closed linear span of its projections, the conclusion follows.

We now turn our attention to the non-commutative case. Let $M$ be the 2 by 2 matrix algebra, generated by two mutually orthogonal, equivalent projections $e$ and $f$ and partial isometries $v$ and $v^{*}$ satisfying

$$
\begin{gathered}
e f=0, v^{*} v=e, v v^{*}=f, v^{2}=0,\left(v^{*}\right)^{2}=0, v e=v, v f=0, \\
f v=v, e v=0, e v^{*}=v^{*}, f v^{*}=0, v^{*} f=v^{*}, v^{*} e=0 .
\end{gathered}
$$

1.5. Lemma. Let $\phi: M \rightarrow B(H)$ be a local representation. Then the operators $\phi(e), \phi(f), \phi(v)$, and $\phi\left(v^{*}\right)$ satisfy the relations

$$
\begin{gathered}
\phi(v)^{2}=\phi\left(v^{*}\right)^{2}=0, \\
\phi(v)=\phi(e) \phi(v)+\phi(v) \phi(e)=\phi(f) \phi(v)+\phi(v) \phi(f), \\
\phi\left(v^{*}\right)=\phi(e) \phi\left(v^{*}\right)+\phi\left(v^{*}\right) \phi(e)=\phi(f) \phi\left(v^{*}\right)+\phi\left(v^{*}\right) \phi(f), \\
\phi(v) \phi(e)=\phi(f) \phi(v) ; \phi(v) \phi(f)=\phi(e) \phi(v), \\
\phi\left(v^{*}\right) \phi(e)=\phi(f) \phi\left(v^{*}\right) ; \phi\left(v^{*}\right) \phi(f)=\phi(e) \phi\left(v^{*}\right), \\
\phi(v) \phi\left(v^{*}\right)+\phi\left(v^{*}\right) \phi(v)=\phi(e)+\phi(f) .
\end{gathered}
$$


Proof. Let $\pi_{v}$ be the representation satisfying $\pi_{v}(v)=\phi(v)$. Then $\phi(v)^{2}=\pi_{v}(v)^{2}=$ $\pi_{v}\left(v^{2}\right)=\pi_{v}(0)=0$. Similarly, $\phi\left(v^{*}\right)^{2}=0$. We apply 1.2 to the idempotents $e+v, f+v, e+v^{*}$, and $f+v^{*}$ :

$$
\begin{gathered}
\phi(e)+\phi(v)=\phi(e+v)=\phi(e+v)^{2}=(\phi(e)+\phi(v))^{2} \\
=\phi(e)^{2}+\phi(v)^{2}+\phi(e) \phi(v)+\phi(v) \phi(e),
\end{gathered}
$$

which implies $\phi(v)=\phi(e) \phi(v)+\phi(v) \phi(e)$. Similar computations show that $\phi(v)=$ $\phi(f) \phi(v)+\phi(v) \phi(f), \phi\left(v^{*}\right)=\phi(e) \phi\left(v^{*}\right)+\phi\left(v^{*}\right) \phi(e)$, and $\phi\left(v^{*}\right)=\phi(f) \phi\left(v^{*}\right)+$ $\phi\left(v^{*}\right) \phi(f)$.

From this it follows that

$$
\begin{aligned}
& \phi(v) \phi(e)=(\phi(f) \phi(v)+\phi(v) \phi(f)) \phi(e)=\phi(f) \phi(v) \phi(e), \\
& \phi(f) \phi(v)=\phi(f)(\phi(e) \phi(v)+\phi(v) \phi(e))=\phi(f) \phi(v) \phi(e) ;
\end{aligned}
$$

hence $\phi(v) \phi(e)=\phi(f) \phi(v)$. Similarly, $\phi(v) \phi(f)=\phi(e) \phi(v), \phi\left(v^{*}\right) \phi(e)=\phi(f) \phi\left(v^{*}\right)$, $\phi\left(v^{*}\right) \phi(f)=\phi(e) \phi\left(v^{*}\right)$.

We now apply 1.2 to the projection $p=\frac{1}{2}\left(e+f+v+v^{*}\right)$ and use the results obtained so far. $\phi(p)=\phi(p)^{2}$ implies

$$
\begin{gathered}
2\left(\phi(e)+\phi(f)+\phi(v)+\phi\left(v^{*}\right)\right)= \\
\phi(e)^{2}+\phi(f)^{2}+\phi(v)^{2}+\phi\left(v^{*}\right)^{2}+\phi(e) \phi(f)+\phi(e) \phi(v)+ \\
\phi(e) \phi\left(v^{*}\right)+\phi(f) \phi(e)+\phi(f) \phi(v)+\phi(f) \phi\left(v^{*}\right)+\phi(v) \phi(e)+ \\
\phi(v) \phi(f)+\phi(v) \phi\left(v^{*}\right)+\phi\left(v^{*}\right) \phi(e)+\phi\left(v^{*}\right) \phi(f)+\phi\left(v^{*}\right) \phi(v), \\
2\left(\phi(e)+\phi(f)+\phi(v)+\phi\left(v^{*}\right)\right)=\phi(e)+\phi(f)+2 \phi(v)+2 \phi\left(v^{*}\right)+ \\
\phi(v) \phi\left(v^{*}\right)+\phi\left(v^{*}\right) \phi(v), \\
\phi(v) \phi\left(v^{*}\right)+\phi\left(v^{*}\right) \phi(v)=\phi(e)+\phi(f) .
\end{gathered}
$$

1.6. Lemma. If $S, T \in M$ and $S T=T S$, then $\phi(S T)=\phi(S) \phi(T)$.

Proof. Let $S=a_{1} e+b_{1} v^{*}+c_{1} v+d_{1} f$ and $T=a_{2} e+b_{2} v^{*}+c_{2} v+d_{2} f$. Then $S T=\left(a_{1} a_{2}+b_{1} c_{2}\right) e+\left(a_{1} b_{2}+b_{1} d_{2}\right) v^{*}+\left(c_{1} a_{2}+d_{1} c_{2}\right) v+\left(c_{1} b_{2}+d_{1} d_{2}\right) f$ and $T S=\left(a_{2} a_{1}+b_{2} c_{1}\right) e+\left(a_{2} b_{1}+b_{2} d_{1}\right) v^{*}+\left(c_{2} a_{1}+d_{2} c_{1}\right) v+\left(c_{2} b_{1}+d_{2} d_{1}\right) f$.

$$
\begin{gathered}
\phi(S) \phi(T)=a_{1} a_{2} \phi(e)+a_{1} b_{2} \phi(e) \phi\left(v^{*}\right)+a_{1} c_{2} \phi(e) \phi(v)+ \\
b_{1} a_{2} \phi\left(v^{*}\right) \phi(e)+b_{1} c_{2} \phi\left(v^{*}\right) \phi(v)+b_{1} d_{2} \phi\left(v^{*}\right) \phi(f)+c_{1} a_{2} \phi(v) \phi(e)+c_{1} b_{2} \phi(v) \phi\left(v^{*}\right)+ \\
c_{1} d_{2} \phi(v) \phi(f)+d_{1} b_{2} \phi(f) \phi\left(v^{*}\right)+d_{1} c_{2} \phi(f) \phi(v)+d_{1} d_{2} \phi(f) .
\end{gathered}
$$

$S T=T S$ implies $b_{1} c_{2}=b_{2} c_{1}, a_{1} b_{2}+b_{1} d_{2}=a_{2} b_{1}+b_{2} d_{1}$, and $a_{2} c_{1}+c_{2} d_{1}=$ $a_{1} c_{2}+c_{1} d_{2}$. Hence, by using the relations 1.5 , we obtain

$$
\begin{gathered}
\phi(S) \phi(T)=\left(a_{1} a_{2}+b_{1} c_{2}\right) \phi(e)+\left(b_{2} c_{1}+d_{1} d_{2}\right) \phi(f)+\left(a_{1} b_{2}+b_{1} d_{2}\right) \phi\left(v^{*}\right)+ \\
\left(a_{2} c_{1}+c_{2} d_{1}\right) \phi(v)=\phi(S T) .
\end{gathered}
$$




\section{A Counterexample}

The computations in section 1 and the obstructions therein suggest the following example of a local representation of the algebra of 2 by 2 matrices, which fails to be a representation. Define $\phi: \mathbf{M}_{2}(\mathbf{C}) \rightarrow B\left(\mathbf{C}^{4}\right)$ by

$$
\phi\left(\left(\begin{array}{ll}
a & b \\
c & d
\end{array}\right)\right)=\left(\begin{array}{llll}
a & 0 & b & 0 \\
0 & a & 0 & c \\
c & 0 & d & 0 \\
0 & b & 0 & d
\end{array}\right) .
$$

For $X=\left(\begin{array}{ll}1 & 0 \\ 0 & 0\end{array}\right)$ and $Y=\left(\begin{array}{ll}1 & 1 \\ 0 & 0\end{array}\right)$, it is easy to check that $\phi(X Y) \neq$ $\phi(X) \phi(Y)$, so $\phi$ is not a representation.

To show that $\phi$ is a local representation, it is enough to prove that $I_{2} \otimes X$ and $\phi(X)$ are similar for all $X \in \mathbf{M}_{2}(\mathbf{C})$. This is a routine exercise in linear algebra: two square matrices $A$ and $B$ are similar if and only if they have the same canonical

Jordan form. Equivalently, they have the same characteristic polynomial and, for every common eigenvalue $\lambda$, the matrices $\lambda I-A$ and $\lambda I-B$ have the same rank.

$$
\text { If } X=\left(\begin{array}{ll}
a & b \\
c & d
\end{array}\right) \text {, then }\left(\begin{array}{cccc}
a & 0 & b & 0 \\
0 & a & 0 & b \\
c & 0 & d & 0 \\
0 & c & 0 & d
\end{array}\right) \text { and }\left(\begin{array}{cccc}
a & 0 & b & 0 \\
0 & a & 0 & c \\
c & 0 & d & 0 \\
0 & b & 0 & d
\end{array}\right) \text { have the same }
$$

characteristic polynomial $P(\lambda)=\left(\lambda^{2}-(a+d) \lambda+a d-b c\right)^{2}$. Note that, for $t \in \mathbf{C}$, $X$ and $Y$ are similar if and only if $X+t I$ and $Y+t I$ are similar. We distinguish several cases:

(1) If $a=d$, subtract $a I$. Then the operators

$$
\left(\begin{array}{cccc}
0 & 0 & b & 0 \\
0 & 0 & 0 & b \\
c & 0 & 0 & 0 \\
0 & c & 0 & 0
\end{array}\right) \text { and }\left(\begin{array}{cccc}
0 & 0 & b & 0 \\
0 & 0 & 0 & c \\
c & 0 & 0 & 0 \\
0 & b & 0 & 0
\end{array}\right)
$$

are unitary equivalent via the unitary

$$
\left(\begin{array}{llll}
1 & 0 & 0 & 0 \\
0 & 0 & 0 & 1 \\
0 & 0 & 1 & 0 \\
0 & 1 & 0 & 0
\end{array}\right)
$$

(2) If $a \neq d$, we may assume (by subtracting $d I$ and dividing by $a-d$ ) that $a=1$ and $d=0$, and thus reduce the problem to showing that

$$
A=\left(\begin{array}{cccc}
1 & 0 & b & 0 \\
0 & 1 & 0 & b \\
c & 0 & 0 & 0 \\
0 & c & 0 & 0
\end{array}\right) \text { and } B=\left(\begin{array}{cccc}
1 & 0 & b & 0 \\
0 & 1 & 0 & c \\
c & 0 & 0 & 0 \\
0 & b & 0 & 0
\end{array}\right) \text { are similar. }
$$

(3) If $b=c=0$, there is nothing to prove.

(4) If one of $b$ and $c$ is 0 , say $c=0, b \neq 0$, then both matrices have characteristic polynomial $P(\lambda)=(\lambda(\lambda-1))^{2}$. Similarity follows once we prove that $\lambda I-A$ and $\lambda I-B$ have the same rank, $\lambda=0,1$. 


$$
\begin{aligned}
& \text { If } \lambda=0 \text {, both }\left(\begin{array}{cccc}
1 & 0 & b & 0 \\
0 & 1 & 0 & b \\
0 & 0 & 0 & 0 \\
0 & 0 & 0 & 0
\end{array}\right) \text { and }\left(\begin{array}{cccc}
1 & 0 & b & 0 \\
0 & 1 & 0 & 0 \\
0 & 0 & 0 & 0 \\
0 & b & 0 & 0
\end{array}\right) \text { have rank 2. If } \\
& \lambda=1 \text {, both }\left(\begin{array}{cccc}
0 & 0 & -b & 0 \\
0 & 0 & 0 & -b \\
0 & 0 & 1 & 0 \\
0 & 0 & 0 & 1
\end{array}\right) \text { and }\left(\begin{array}{cccc}
0 & 0 & -b & 0 \\
0 & 0 & 0 & 0 \\
0 & 0 & 1 & 0 \\
0 & -b & 0 & 1
\end{array}\right) \text { have rank } 2 \text {. }
\end{aligned}
$$

(5) If $b \neq 0, c \neq 0$, then $I_{2} \otimes X$ and $\phi(X)$ have the same characteristic polynomial $P(\lambda)=(\lambda(\lambda-1)-b c)^{2}$. If $P(\lambda)=0$, then $\lambda \neq 0,1$, and elementary row operations show that $\left(\begin{array}{cccc}\lambda-1 & 0 & -b & 0 \\ 0 & \lambda-1 & 0 & -b \\ -c & 0 & \lambda & 0 \\ 0 & -c & 0 & \lambda\end{array}\right)$ has the same rank $\operatorname{as}\left(\begin{array}{cccc}\lambda(\lambda-1) & 0 & -\lambda b & 0 \\ 0 & \lambda(\lambda-1) & 0 & -\lambda b \\ 0 & 0 & 0 & 0 \\ 0 & 0 & 0 & 0\end{array}\right)$, which is 2. In the same fashion, $\left(\begin{array}{cccc}\lambda-1 & 0 & -b & 0 \\ 0 & \lambda-1 & 0 & -c \\ -c & 0 & \lambda & 0 \\ 0 & -b & 0 & \lambda\end{array}\right)$ and $\left(\begin{array}{cccc}\lambda(\lambda-1) & 0 & -\lambda b & 0 \\ 0 & \lambda(\lambda-1) & 0 & -\lambda c \\ 0 & 0 & 0 & 0 \\ 0 & 0 & 0 & 0\end{array}\right)$

have rank 2. This completes the proof.

\section{2-LOCAL REPRESENTATIONS}

Having obtained as much as possible from 1-local representations, we now address the question of whether the 2-local property yields any additional information.

3.1. Definition. A bounded, linear map $\phi: M \rightarrow B(H)$ is called a 2-local representation if, for every $x, y \in M$, there exists a bounded representation $\pi_{x, y}: M \rightarrow$ $B(H)$ such that $\phi(x)=\pi_{x, y}(x)$ and $\phi(y)=\pi_{x, y}(y)$.

3.2. Remark. Let $\phi: M \rightarrow B(H)$ be a 2-local representation and $x, y \in M$. If $x y=0$, then $\phi(x) \phi(y)=0$.

Proof. $\phi(x) \phi(y)=\pi_{x, y}(x) \pi_{x, y}(y)=\pi_{x, y}(x y)=0$.

3.3. The above property allows us to complete relations 1.5. If $M=\mathbf{M}_{2}(\mathbf{C})$ and if $\phi: M \rightarrow B(H)$ is a 2-local representation, then the relations immediately preceding Lemma 1.5 show that

$$
\phi(v) \phi(f)=\phi(e) \phi(v)=\phi(f) \phi\left(v^{*}\right)=\phi\left(v^{*}\right) \phi(e)=0 .
$$

Relations 1.5 become

$$
\begin{gathered}
\phi(v)=\phi(v) \phi(e)=\phi(f) \phi(v), \\
\phi\left(v^{*}\right)=\phi(e) \phi\left(v^{*}\right)=\phi\left(v^{*}\right) \phi(f), \\
\phi\left(v^{*}\right) \phi(v)=\phi(e), \phi(v) \phi\left(v^{*}\right)=\phi(f) .
\end{gathered}
$$


We need to prove only the last two. From the first two and 1.5 we have

$$
\begin{gathered}
\phi\left(v^{*}\right) \phi(v)=\phi(e) \phi\left(v^{*}\right) \phi(v) \phi(e) \\
=\phi(e)\left(\phi(e)+\phi(f)-\phi(v) \phi\left(v^{*}\right)\right) \phi(e) \\
=\phi(e)+\phi(e) \phi(f) \phi(e)-\phi(e) \phi(v) \phi\left(v^{*}\right) \phi(e)=\phi(e) .
\end{gathered}
$$

A similar argument establishes $\phi(v) \phi\left(v^{*}\right)=\phi(f)$.

3.4. Proposition. Every 2-local representation $\phi: \mathbf{M}_{2}(\mathbf{C}) \rightarrow B(H)$ is a representation.

Proof. We use the notation in 1.6. Recall that $S=a_{1} e+b_{1} v^{*}+c_{1} v+d_{1} f, T=$ $a_{2} e+b_{2} v^{*}+c_{2} v+d_{2} f$ and $S T=\left(a_{1} a_{2}+b_{1} c_{2}\right) e+\left(a_{1} b_{2}+b_{1} d_{2}\right) v^{*}+\left(c_{1} a_{2}+d_{1} c_{2}\right) v+$ $\left(c_{1} b_{2}+d_{1} d_{2}\right) f$. Relations 3.3, applied to 1.6, lead to

$$
\begin{gathered}
\phi(S) \phi(T)=a_{1} a_{2} \phi(e)+a_{1} b_{2} \phi(e) \phi\left(v^{*}\right)+a_{1} c_{2} \phi(e) \phi(v)+ \\
b_{1} a_{2} \phi\left(v^{*}\right) \phi(e)+b_{1} c_{2} \phi\left(v^{*}\right) \phi(v)+b_{1} d_{2} \phi\left(v^{*}\right) \phi(f)+c_{1} a_{2} \phi(v) \phi(e)+c_{1} b_{2} \phi(v) \phi\left(v^{*}\right)+ \\
c_{1} d_{2} \phi(v) \phi(f)+d_{1} b_{2} \phi(f) \phi\left(v^{*}\right)+d_{1} c_{2} \phi(f) \phi(v)+d_{1} d_{2} \phi(f) \\
=a_{1} a_{2} \phi(e)+a_{1} b_{2} \phi\left(v^{*}\right)+b_{1} c_{2} \phi(e)+b_{1} d_{2} \phi\left(v^{*}\right)+c_{1} a_{2} \phi(v)+ \\
c_{1} b_{2} \phi(f)+d_{1} c_{2} \phi(v)+d_{1} d_{2} \phi(f) \\
=\left(a_{1} a_{2}+b_{1} c_{2}\right) \phi(e)+\left(a_{1} b_{2}+b_{1} d_{2}\right) \phi\left(v^{*}\right)+\left(c_{1} a_{2}+d_{1} c_{2}\right) \phi(v)+ \\
\left(c_{1} b_{2}+d_{1} d_{2}\right) \phi(f)=\phi(S T) .
\end{gathered}
$$

3.5. Corollary. Let $M$ be the direct sum of an abelian von Neumann algebra, and a type $\mathrm{I}_{2}$ factor. Then every 2-local representation $\phi: M \rightarrow B(H)$ is multiplicative.

Proof. Let $e$ be the central projection in $M$ such that $M(I-e)$ is isomorphic to $\mathbf{M}_{2}(\mathbf{C})$ and $M e$ is abelian. If $x, y \in M$, then $x=a_{1}+x_{1}$ and $y=a_{2}+x_{2}$, where $a_{i} \in M e, x_{i} \in M(I-e), i=1,2$. Since $\phi\left(a_{1} a_{2}\right)=\phi\left(a_{1}\right) \phi\left(a_{2}\right), \phi\left(x_{1} x_{2}\right)=$ $\phi\left(x_{1}\right) \phi\left(x_{2}\right), \phi\left(a_{i}\right)=\phi(e) \phi\left(a_{i}\right) \phi(e), \phi\left(x_{i}\right)=\phi(f) \phi\left(x_{i}\right) \phi(f), i=1,2$, we get $\phi(x) \phi(y)=\phi(x y)$.

3.6. Corollary. Let $M$ be a von Neumann algebra, and let $\phi: M \rightarrow B(H)$ be a 2-local representation. Then $\phi$ is a representation.

Proof. The von Neumann algebra generated by any two projections $e$ and $f$ in $M$ is either abelian, of type $\mathrm{I}_{2}$, or the direct sum of an abelian and a type $\mathrm{I}_{2}$ ( $[9]$, 12.4.11). From 1.4, 3.4 and 3.5 we infer that $\phi(e f)=\phi(e) \phi(f)$. Since $M$ equals the norm-closed linear span of its projections, $\phi$ is multiplicative on $M$.

We conclude with some remarks on 2-local automorphisms of von Neumann algebras. From [11], [2], 17] we know that every 2-local automorphism of $B(H)$ ( $H$ separable) is an automorphism. In fact, no linearity, surjectivity or continuity are assumed. We generalize this result, in the context of linear maps.

3.7. Definition. Let $M$ be a von Neumann algebra. A bounded, linear map $\phi$ : $M \rightarrow M$ is called a 2-local automorphism of $M$ if, for every $x, y \in M$, there exists a bounded, linear (not necessarily self-adjoint) automorphism $\pi_{x, y}$ of $M$ such that $\phi(x)=\pi_{x, y}(x)$ and $\phi(y)=\pi_{x, y}(y)$. 
Recall that a von Neumann algebra $M$ is singly generated if there is an operator $S \in M$ such that $M$ equals the ultraweak closure of the algebra generated by $I, S$, and $S^{*}$.

3.8. Proposition. Let $M$ be a singly generated von Neumann algebra. Then every ultraweakly continuous 2-local automorphism of $M$ is an automorphism.

Proof. Let $\phi: M \rightarrow M$ be an ultraweakly continuous 2-local automorphism. Then, by $3.6, \phi$ is a representation. Let $\pi_{I}$ be the automorphism of $M$ such that $\pi_{I}(I)=$ $\phi(I)$. Then $\pi_{I}(I)=I$; hence $\phi(I)=I$. If $\phi(x)=0$, then $\phi(x)=\pi_{x}(x)$ for some automorphism $\pi_{x}$. This shows that $x=0$, so $\phi$ is injective. If $S$ is the generator of $M$, there exists an automorphism $\pi=\pi_{S}$ of $M$ such that $\phi(S)=\pi(S)$ and $\phi\left(S^{*}\right)=\pi\left(S^{*}\right)$. Then $\phi$ and $\pi$ are equal on the algebra generated by $I, S$, and $S^{*}$. It is well known that $\pi$ is ultraweakly continuous. In addition, ultraweak continuity of $\phi$ shows that the range of $\phi$ must be the entire $M$, which concludes the proof.

3.9. The class of singly generated von Neumann algebras contains all type I algebras [14] and all properly infinite ones [18. While it is still unknown whether every finite von Neumann algebra is singly generated, the answer is affirmative for at least three important classes: finite algebras containing Cartan subalgebras [15], type $\mathrm{II}_{1}$ factors with property $\Gamma$ and tensor products of type $\mathrm{II}_{1}$ factors $[5]$.

\section{REFERENCES}

1. M. Brešar and P. Šemrl, Mappings which preserve idempotents, local automorphisms, and local derivations, Canad. J. Math. 45 (1993), 483-496. MR 1222512 (94k:47054)

2. M. Brešar and P. Šemrl, On local automorphisms and mappings that preserve idempotents, Studia Math. 113 (1995), 101-108. MR 1318418 (96i:47058)

3. R. Crist, Local derivations on operator algebras, J. Funct. Anal. 135 (1996), 76-92. MR 1367625 (96m:46128)

4. R. Crist, Local automorphisms, Proc. Amer. Math. Soc. 128 (2000), 1409-1415. MR 1657786 (2001a:47078)

5. L. Ge and S. Popa, On some decomposition properties of factors of type $\mathrm{II}_{1}$, Duke Math. J. 94 (1998), 79-101. MR 1635904 (99j:46070)

6. D. Han and S. Wei, Local derivations of nest algebras, Proc. Amer. Math. Soc. 123 (1995), 3095-3100. MR 1246521 (95m:47077)

7. R. V. Kadison, Derivations of operator algebras, Ann. of Math. 83 (1966), 280-293. MR 0193527 (33:1747)

8. R. V. Kadison, Local derivations, J. Algebra 130 (1990), 494-509. MR 1051316 (91f:46092)

9. R. V. Kadison and J. R Ringrose, Fundamentals of the theory of operator algebras, vol. II, Academic Press, 1986. MR 0859186 (88d:46106)

10. E. Kirchberg, The derivation and the similarity problem are equivalent, J. Operator Theory. 36 (1996), 59-62. MR 1417186 (97f:46108)

11. D. R. Larson and A. R. Sourour, Local derivations and local automorphisms of $\mathcal{B}(X)$, Proc. Sympos. Pure Math. 51, Part 2, Providence, Rhode Island 1990, pp. 187-194. MR 1077437 (91k:47106)

12. L. Molnar, Local automorphisms of operator algebras on Banach spaces, Proc. Amer. Math. Soc. 131 (2003), 1867-1874. MR 1955275 (2003j:47050)

13. L. Molnar and P. Šemrl, Local automorphisms of the unitary group and the general linear group on a Hilbert space, Expo. Math. 18 (2000), 231-238. MR 1763889 (2001a:47083)

14. C. Pearcy, $W^{*}$-algebras with a single generator, Proc. Amer. Math. Soc. 13 (1962), 831-832. MR 0152904 (27:2875)

15. S. Popa, Notes on Cartan subalgebras in type $\mathrm{II}_{1}$ factors, Math. Scand. 57 (1985), 171-188. MR 0815434 (87f:46114)

16. S. Sakai, Derivations of $W^{*}$-algebras, Ann. of Math. 83 (1966), 273-279. MR $0193528(33: 1748)$ 
17. P. Šemrl, Local automorphisms and derivations on $\mathcal{B}(H)$, Proc. Amer. Math. Soc. 125 (1997), 2677-2680. MR 1415338 (98e:46082)

18. W. Wogen, On generators for von Neumann algebras, Bull. Amer. Math. Soc. 75 (1969), 95-99. MR 0236725 (38:5020)

Department of Mathematics and Computer Science, Wagner College, Staten Island, NeW YorK 10301

E-mail address: fpop@wagner.edu 